\title{
Research on Greenhouse Intelligence Acquisition Technology Based on Wireless Data Transmission System in the Background of Big Data
}

\author{
Chang Su, a Chao Yang ${ }^{1, b}$ and Hongbo Chu ${ }^{1, c}$ \\ ${ }^{1}$ Heilongjiang Unigversity of Technology, Heilongjiang, Jixi , 158100 \\ ajxdxsc@163.com, bjxdxyc@163.com
} Keywords: Greenhouse Intelligence Acquisition Technology, Wireless Data Transmission System,
Big Data

\begin{abstract}
In view of the current problems of insufficient capacity for agricultural facilities management and environmental monitoring, insufficient accumulation of relevant data for agricultural production, and low level of intellectualization of agricultural production, the greenhouse greenhouses were targeted for the application of the Internet of Things technology to design a real-time greenhouse temperature and humidity. Environmental information such as soil temperature and humidity, light, etc., and access to the Internet cloud control platform or mobile client through WIFI technology for data communication, real-time collection, display, storage and sharing of environmental data, and analysis of the collected data Judgment, automatic control of sprinkler motors and heating equipment intelligent greenhouse system. Experiments show that the system has the characteristics of simple installation, friendly interface, strong practicality, and easy extension. Android client and We-Chat public account realize the remote mobility management of the system. Good data interface helps big data collection and analysis, and can adapt to intelligence. Big data application needs in agriculture.

Agricultural big data is a comprehensive application of big data technology, ideas and methods in the entire agricultural production and sales process. Its development has driven the development of agricultural informationization, and has continuously promoted the optimization of agricultural economy and the construction of smart agriculture. Although the importance of agricultural big data has gradually emerged, the research on agricultural big data still focuses on the analysis and processing of data. As the most important gathering link in the value mining of big agricultural data, it is still relatively weak.
\end{abstract}

In recent years, the research on the collection of agricultural environmental information has developed rapidly. ZigBee networks and GPRS networks have been widely used, and most agricultural environmental monitoring systems are equipped with independent upper computers or mobile clients. Yu Pan and others proposed the ZigBee wireless sensor network combined with 3G network remote farm environment monitoring system. Zhao Wenbing and others proposed a long-range greenhouse monitoring system that combines ZigBee network, WIFI network and Android client. Han Liying also conducted this issue. The research has achieved certain results.

Based on the rapid development of Internet technology, the increasing popularity of Internet of Things technology, and the growing maturity of cloud computing technology, the use of cost-effective single-chip microcomputers combined with environmental sensors to form an environmental monitoring module, real-time collection of environmental data for agricultural facilities; using WIFI technology to access the Internet cloud control platform Real-time collection and processing of data; real-time display of data through the mobile client, control of the implementation of the adjustment of environmental parameters, to form a closed-loop intelligent agricultural facilities system from information monitoring to information display and action control.

In the management system of greenhouses, the temperature, humidity, carbon dioxide content, light intensity, and soil conditions inside the greenhouse must be mastered in real time, which can effectively ensure the growth of the internal crops in a good environment. At present, more greenhouses use different equipment to ensure the growth of good crops. For example: ventilation systems, external shading, heaters, etc. However, these devices require manual real-time monitoring operations, and there are many inconveniences. At present, in order to provide a better growing 
environment for crops, the various parameters in the greenhouse are accurately controlled, and a smart greenhouse management and control system is gradually established to implement effective intelligent adjustment of various parameters for automated management.

\section{Analysis on System Framework}

The greenhouse intelligent monitoring system is mainly divided into three components: the on-site monitoring subsystem of the greenhouse, the remote monitoring subsystem of the greenhouse, and the database. These three parts are mutually independent, and the operability of each subsystem is with good scalability, the specific design of the two subsystems is as follows:

The design basis of the site monitoring subsystem is the C/S architecture. It is based on the site monitoring computer, installs the monitoring software on the site monitoring computer, distributes the CAN bus in the greenhouse, and adopts the CANOpen protocol. Through this protocol and the distributed CAN bus Collect greenhouse-related data and related communications of the control system to achieve the collection of environmental factors in the greenhouse, and effectively control greenhouse environmental control equipment. There are two modules in the site monitoring computer monitoring software, one is the output module of greenhouse environmental data information, and the other is the input module of greenhouse environmental data information [1]. First, the input module: the sensor can collect environmental information of the greenhouse. The environmental information in the greenhouse mainly includes the humidity, temperature and solar radiation intensity in the greenhouse, and the ambient temperature information mainly includes air temperature, wind direction, humidity, light intensity and wind speed. The input module will obtain this information through the sensor and send it to the computer through the CAN bus distributed on site. The greenhouse site monitoring computer will collect this information and display it through a certain conversion in a specific numerical manner. This information is stored in the database; second, the output module: the CAN bus distributed in the greenhouse can transmit the control signal sent from the monitoring software running on the computer to the output module, and the output module can control the relay to perform related actions according to the control signal and command. In order to achieve the regulation of the greenhouse environment, such as fan, shade net, air conditioning and other equipment to adjust the temperature and humidity, solar radiation intensity. In addition to the on-site adjustment of the greenhouse environment, the on-site monitoring subsystem can also query and export relevant data, perform video surveillance on the greenhouse, and coordinate the control of the relevant equipment status with the remote monitoring system.

The design basis of the remote monitoring subsystem is the $\mathrm{B} / \mathrm{S}$ architecture, which is divided into three components. First, the data acquisition layer: its main function is to obtain information related to the greenhouse environment; second, the logical application layer: its main function is to issue relevant control commands for the greenhouse environment; and third, the interactive layer: its main function is to provide interactions. Sexual control logic to remotely acquire, monitor and control greenhouse environmental information.

The remote monitoring subsystem has real-time and off-site viewing functions of greenhouse environmental information, remote control of equipment, and remote video monitoring of greenhouses. Even if managers or researchers are located in different places, they can understand the status of the greenhouse environment in real time and take corresponding monitoring measures, breaking the geographical, spatial and temporal limits of greenhouse monitoring [2].

\section{Research on Greenhouse Intelligent Acquisition Technology}

The intelligent greenhouse system is a resource-saving and high-efficiency agriculture development technology. It is developed on the basis of an ordinary solar greenhouse, combined with modern computer automation technology, intelligent sensing technology and other high-tech means. The control level of the greenhouse environment is one of the important technical conditions that determine the intelligent greenhouse system. Foreign countries have relatively early research on 
greenhouse environmental control technologies and relatively mature technologies. From the successful experiences of developed countries such as the Netherlands and Israel, it is found that the use of environmental automatic control technologies is an effective means to improve greenhouse environmental control technologies. The domestic research on greenhouse environmental control technologies started late. This paper researched and designed an intelligent greenhouse control system [3]. The intelligent collector of this system uses Cygnal's C8051F040 single-chip microcomputer and is supplemented by corresponding circuits through various high performances. The sensor realizes real-time data collection such as temperature, humidity and light in the greenhouse, and sends the collected data to the intelligent processor through the bus.

The intelligent collector mainly includes a power circuit, a CPU circuit, a CAN interface circuit, an RS-232 interface circuit, an E2 PROM memory, three temperature sensors, and three humidity sensors, and one light sensor. The value of the environmental factor parameter at each point in the greenhouse is different. Therefore, it is necessary to arrange 10 intelligent collectors in parallel to collect the temperature, humidity, and illuminance at different points.

\section{Hardware Components of the Smart Collector}

The CPU used in this system is Cygnal's C8051F040 microcontroller [4]. CygnalC8051F series MCU is an integrated mixed-signal system-on-chip (SoC) systemonchip, using Cygnal's patented CIP-51 microcontroller core; CIP-51 and The MCS-51TM instruction set is fully compatible and can use standard $803 \mathrm{x} / 805 \mathrm{x}$ assemblers and compilers for software development; it is easy to program and use; the CIP-51 uses a pipelined architecture and has a large instruction execution speed compared to the standard 8051 architecture. Increase; In a standard 8051, all instructions except MUL and DIV require 12 or 24 system clock cycles; for the CIP-51 core, $70 \%$ of instructions execute in 1 or 2 system clock cycles. The execution time of the four instructions is greater than four system clock cycles. The high-speed running kernel is beneficial to the real-time operation and processing functions of the system. On-chip resources are abundant, which helps to reduce power consumption and greatly reduce the board size.

Due to the abundant resources on the chip, we can use complex peripheral devices to implement complex system functions. This system maximizes the use of on-chip resources. They are implemented entirely in hardware and can generate interrupts to the CPU. Therefore, CPU intervention is rarely required, making the entire design simple and practical.

The CPU circuit of this system is specifically described as follows:

The C8051F040 has on-chip JTAG and debug circuitry to enable non-intrusive, full-speed on-line system debugging. Supports observation and modification of memory and registers, support for breakpoints, watchpoints, stack pointers, and single-step execution. No additional target RAM, program memory, timers, or communication channels are required for debugging, and all analog and digital peripherals operate normally. When the MCU steps or stops running when a breakpoint is encountered, all peripherals stop running to maintain synchronization.

The analog power supply and the digital power supply and the analog ground and digital ground are connected with a single point of zero-ohm resistor. The zero-ohm resistor is equivalent to a very narrow current path, which can effectively limit the loop current and suppress the noise. The resistance is attenuated across all frequency bands (zero-ohm resistors also have impedance).

The voltage reference of the analog signal uses the internal voltage reference of the microcontroller. This voltage reference consists of a $1.2 \mathrm{~V}, 15 \times 10-6 /{ }^{\circ} \mathrm{C}$ (typ) bandgap voltage reference generator and a 2 -fold gain output buffer amplifier.

Use the built-in watchdog of C8051F040 to make the program reset to normal operation after running under strong interference.

In the CAN bus interface circuit, the controller selects the integrated CAN controller in the C8051F040 as the BoschCAN controller. In order to improve the anti-interference performance of the bus, the system uses DCDC isolated power supply circuit and high-speed digital optocoupler to completely isolate the CAN bus controller and transceiver.

The serial communication interface circuit can realize the function of mutual communication 
with the serial port of the host computer, and the device initialization parameters can be configured through this interface. Use C8051F040 full-duplex hardware UART0 to achieve serial communication, because C8051F040's working voltage is $3 \mathrm{~V}$, itself with a serial communication port, but its level is TTL level, need to be converted to RS-232 standard level before Communication with the PC. Therefore, the serial communication interface uses the SP3223 chip. The SP3223 operates from 3.3 to $5.0 \mathrm{~V}$ and uses a $0.1 \mu \mathrm{F}$ capacitor to generate an internally-efficient charge pump that generates the RS232 level standard [5].

The T24C04 is an I2C-interfaced E2 PROM device. It has a 512-byte capacity and can erase/write 1 million times per byte. The data retention time is more than 40 years. It has an automatic erase function when writing, and also has a page write function, which can write 16 bytes at a time. In the smart collector, parameters for configuring the smart collector are saved. When the program is initialized, the smart collector is preset according to these parameters.

The sensor for measuring humidity signal adopts S302H3 humidity acquisition module, and the output voltage is 1 to $5 \mathrm{VDC}$; the sensor for measuring the light intensity signal adopts EL7900 current output type light sensor, and the output is connected to $2 \mathrm{k} \Psi$ resistance, and is converted to 0 to 3 VDC output; the opening degree sensor Potentiometric sensors are used and the output voltage is 1 to 5 VDC. Since these signals can be converted into DC voltage signals, only the buffer amplifier circuit can be used in the processing circuit to meet the needs of subsequent circuits. The analog signal adjusts the gain of the signal through the operational amplifier LM358 so that it meets the on-chip AD sampling requirement of the single-chip C8051F040. Humidity signal and light intensity signal adopt on-chip 12-bit $\mathrm{AD}$ acquisition, and opening degree adopts on-chip 8-bit $\mathrm{AD}$ acquisition.

The temperature sensor adopts the DS18B20 digital temperature sensor of "one-wire bus interface", connects the data line to the I/O port of the microcontroller, and acquires the temperature sensor value on the bus through software communication. The wiring is simple and convenient.

\section{Conclusion}

During the research process, on the basis of fuzzy control technology, the use of relatively new and stable electronic components and technical standards, the use of automated professional knowledge to study and design a smart greenhouse control system to achieve the temperature in the greenhouse, Comprehensive control of humidity, illuminance, etc. Based on the intelligent collector realized by Cygnal's C8051F040 single-chip microcomputer, supplemented by corresponding circuits, real-time data collection functions such as temperature, humidity, and illumination in the greenhouse are realized through various high-performance sensors, ensuring the real-time data collection and accuracy.

\section{References}

[1] Zhang Yaxin, Guo Aimin. Design of push-pull air-discharging mechanism in greenhouses [J]. Journal of Chifeng University(Natural Science). 2013 (01)

[2] Xu Yuemin. Technical Research on Urban Lighting District [J]. Talented Intelligence. 2012 (32)

[3] Liu Jun. Electromagnetic Compatibility Design of PCB [J]. Petroleum Instruments. 2012 (01)

[4] Xuan Xuan, Meng Future, Lu Mingxiang. Liaoning Province Agricultural Science and Technology Information Technology Integration and Demonstration [J]. Agricultural Network Information. 2011 (04)

[5] Wang Xinkun, Li Hong. Research status and development trend of greenhouses in China [J]. Journal of Drainage and Irrigation Machinery Engineering. 2010 (02)

[6] Peng L, Fang W. Heterogeneity of Inferring Reputation of Cooperative Behaviors for the Prisoners' Dilemma Game [J]. Physica A: Statistical Mechanics and its Applications, 2015, 433: 367-378. 